\title{
Biomechanical study
}

All tests were performed at room temperature $\left(25 \pm 1{ }^{\circ} \mathrm{C}\right)$, and specimens were kept moist with saline solution. A material testing system (MTS Bionix 858, Eden Prairie, MN, USA) with a custom-made clamping device was used for tensile testing (Fig. 1). The clamping device was rigidly mounted onto the plate of the material testing machine, and the peripheral section of the medial meniscus was placed in a mechanical screw action clamp. In order to prevent meniscus slippage, the clamp was equipped with corrugated jaw faces (Fig. 2). To avoid interference with the stiffness analysis, the menisci were clamped $1 \mathrm{~cm}$ medial to the sutures or the TGs of the meniscus. After a preload of $2 \mathrm{~N}$, all specimens were subjected to 1000 cycles of a load between 2 and $20 \mathrm{~N}$ at a rate of $0.5 \mathrm{~Hz}$. Subsequently, specimens were loaded to failure at a rate of $0.5 \mathrm{~mm} / \mathrm{s}$. The number of cycles, displacement, and loads were recorded by MTS software. The following parameters were analyzed in all tests: (1) displacements after 100, 500, and 1000 cycles and (2) the maximum load, stiffness, and elongation at failure load. The displacement was defined as the differences in the crosshead position from the peak of the first cycle to the peak of cycle 100,500 , and 1000. The stiffness was calculated as the steepest slope of the load-deformation curve spanning $30 \%$ of the data points collected between load initiation and the maximum load at failure. Elongation was measured as the total displacement of sutures or grafts at maximum failure load. Additionally, the mode of failure was determined by visual inspection.

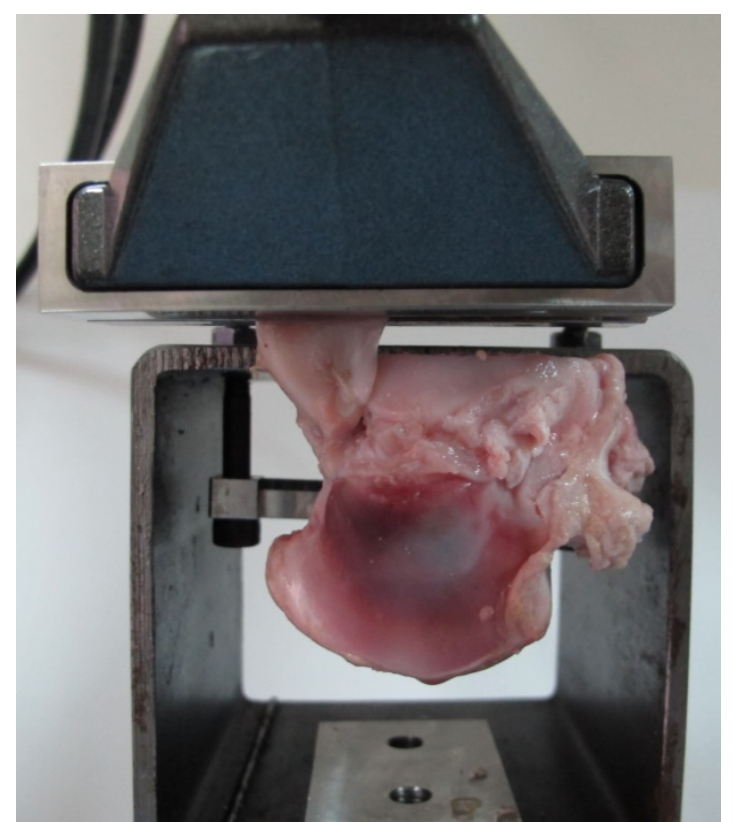

Fig. 1 Biomechanical test setup

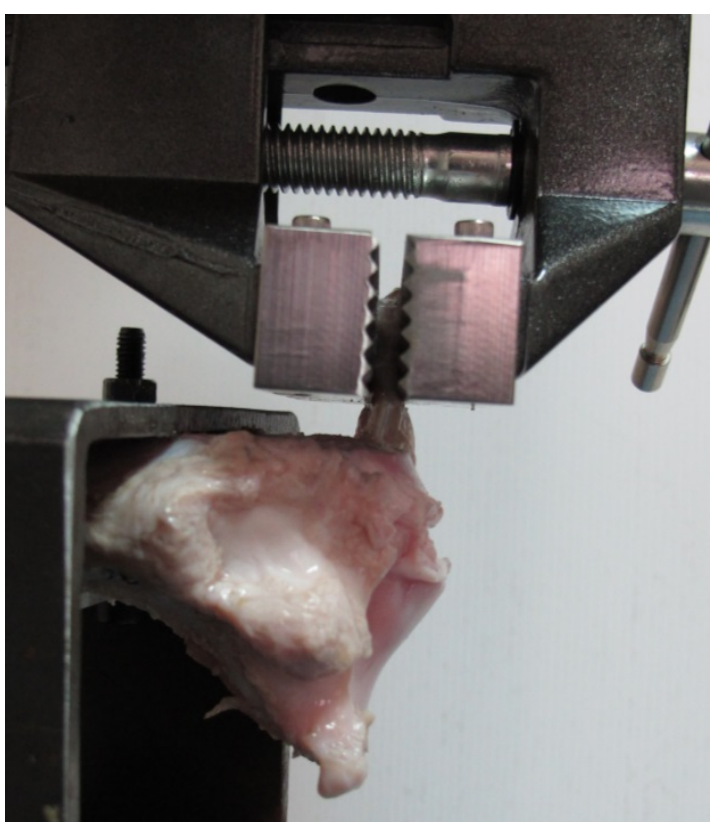

Fig. 2 The clamp was equipped with corrugated jaw faces 


\section{Statistical analysis}

A Mann-Whitney U-test was performed to evaluate group differences in maximum load, stiffness, and elongation at failure load. A Kruskal-Wallis test was used to test group differences in displacements at the three designated loading cycles. The significance level was set to $p<0.05$. For all statistical analyses, SPSS 20.0 (IBM-SPSS, Armonk, NY, USA) was used. The Kolmogorov-Smirnov test was performed to determine if data were normally distributed.

Independent T-test:

significant group differences at Max load, Stiffness, and Displacement at failure.

Group Statistics

\begin{tabular}{|ll|r|r|r|r|}
\hline & Group & $\mathrm{N}$ & \multicolumn{1}{c|}{ Mean } & Std. Deviation & Std. Error Mean \\
\hline \multirow{2}{*}{ MaxLoad } & Suture & 6 & 258.4950 & 44.46535 & 18.15290 \\
& Tendon & 6 & 176.9300 & 46.41853 & 18.95029 \\
& Suture & 6 & 14.9133 & 3.21835 & 1.31389 \\
Stiffness & Tendon & 6 & 26.6217 & 5.62866 & 2.29789 \\
& Suture & 6 & 24.3217 & 2.88527 & 1.17791 \\
\multirow{2}{*}{ Dis_failure } & Tendon & 6 & 14.8117 & 4.03361 & 1.64671 \\
& & &
\end{tabular}

\begin{tabular}{|c|c|c|c|c|c|c|c|c|c|c|}
\hline \multicolumn{11}{|c|}{ Independent Samples Test } \\
\hline & & \multicolumn{2}{|c|}{$\begin{array}{c}\text { Levene's Test for Equality of } \\
\text { Variances }\end{array}$} & \multicolumn{7}{|c|}{ t-test for Equality of Means } \\
\hline & & \multirow[b]{2}{*}{$\mathrm{F}$} & \multirow[b]{2}{*}{ Sig. } & \multirow[b]{2}{*}{$t$} & \multirow[b]{2}{*}{ df } & \multirow[b]{2}{*}{ Sig. (2-tailed) } & \multirow{2}{*}{$\begin{array}{c}\text { Mean } \\
\text { Difference }\end{array}$} & \multirow{2}{*}{$\begin{array}{l}\text { Std. Error } \\
\text { Difference }\end{array}$} & \multicolumn{2}{|c|}{$\begin{array}{c}95 \% \text { Confidence Interval of the } \\
\text { Difference }\end{array}$} \\
\hline & & & & & & & & & Lower & Upper \\
\hline \multirow[t]{2}{*}{ MaxLoad } & $\begin{array}{l}\text { Equal variances } \\
\text { assumed }\end{array}$ & .523 & .486 & 3.108 & 10 & 011 & 81.56500 & 26.24198 & 23.09424 & 140.03576 \\
\hline & $\begin{array}{l}\text { Equal variances not } \\
\text { assumed }\end{array}$ & & & 3.108 & 9.982 & .011 & 81.56500 & 26.24198 & 23.07960 & 140.05040 \\
\hline \multirow[t]{2}{*}{ Stiffness } & $\begin{array}{l}\text { Equal variances } \\
\text { assumed }\end{array}$ & .640 & .442 & -4.423 & 10 & & -11.70833 & 2.64700 & -17.60621 & -5.81045 \\
\hline & $\begin{array}{l}\text { Equal variances not } \\
\text { assumed }\end{array}$ & & & -4.423 & 7.954 & .002 & -11.70833 & 2.64700 & -17.81852 & -5.59814 \\
\hline \multirow[t]{2}{*}{ Dis_failure } & $\begin{array}{l}\text { Equal variances } \\
\text { assumed }\end{array}$ & .270 & .614 & 4.697 & 10 & & 9.51000 & 2.02463 & 4.99884 & 14.02116 \\
\hline & $\begin{array}{l}\text { Equal variances not } \\
\text { assumed }\end{array}$ & & & 4.697 & 9.055 & .001 & 9.51000 & 2.02463 & 4.93421 & 14.08579 \\
\hline
\end{tabular}




\section{Cyclic}

Mixed model ANOVA: no significant group differences was found

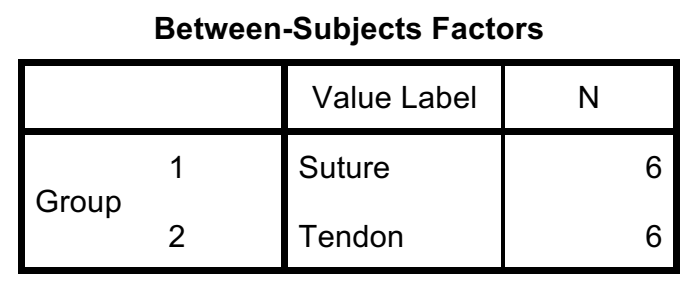

\begin{tabular}{|l|l|r|r|r|}
\hline & Group & \multicolumn{1}{c|}{ Mean } & Std. Deviation & N \\
\hline & Suture & 1.4983 & .48619 & 6 \\
Dis_100cycle & Tendon & .9883 & .52773 & 6 \\
& Total & 1.2433 & .55224 & 12 \\
& Suture & 2.2367 & .60305 & 6 \\
Dis_500cycle & Tendon & 1.9350 & .77053 & 6 \\
& Total & 2.0858 & .67823 & 12 \\
& Suture & 2.6817 & .60829 & 6 \\
& Tendon & 2.4200 & .91686 & 6 \\
Dis_1000cycle & Total & 2.5508 & .75430 & 12 \\
& &
\end{tabular}

\section{Estimates}

Measure: MEASURE_1

\begin{tabular}{|l|r|r|r|r|}
\hline Group & Mean & Std. Error & \multicolumn{2}{|c|}{$95 \%$ Confidence Interval } \\
\cline { 4 - 5 } & & & Lower Bound & Upper Bound \\
\hline Suture & 2.139 & .263 & 1.553 & 2.725 \\
Tendon & 1.781 & .263 & 1.195 & 2.367 \\
\hline
\end{tabular}

Pairwise Comparisons

Measure: MEASURE_1

\begin{tabular}{|c|c|c|c|c|c|c|}
\hline \multirow[t]{2}{*}{ (I) Group } & \multirow[t]{2}{*}{ (J) Group } & \multirow[t]{2}{*}{$\begin{array}{c}\text { Mean } \\
\text { Difference (I-J) }\end{array}$} & \multirow[t]{2}{*}{ Std. Error } & \multirow[t]{2}{*}{ Sig. ${ }^{a}$} & \multicolumn{2}{|c|}{$\begin{array}{c}\text { 95\% Confidence Interval for } \\
\text { Difference }^{a}\end{array}$} \\
\hline & & & & & Lower Bound & Upper Bound \\
\hline Suture & Tendon & .358 & .372 & .359 & -.471 & 1.186 \\
\hline Tendon & Suture & -.358 & .372 & .359 & -1.186 & .471 \\
\hline
\end{tabular}

Based on estimated marginal means 